\title{
A generalization of a problem of Chebyshev
}

\author{
by \\ JürGEN G. Hinz (Marburg)
}

1. The interest of several mathematicians has been attracted by Chebyshev's classical result to the effect that the greatest prime factor of $\prod_{l \leq x}\left(l^{2}+1\right)$ tends to infinity faster than any constant multiple of $x$.

Let us turn our attention to the more general question concerning the order of magnitude of the largest prime factor $P_{x}$ of $\prod_{l<x} f(l)$, where $f$ is any irreducible polynomial of degree greater than 1 with integer coefficients. T. Nagell was the first to give a non-trivial lower bound for $P_{x}$. He proved in [6] that for any $a<1$ and for all sufficiently large $x$,

$$
P_{x}>x(\log x)^{a} .
$$

No further progress was made with this problem until P. Erdös ([1]) in 1952 introduced a new method which increased the lower bound in (1) to $x \exp \left\{c(f) \log _{2} x \cdot \log _{3} x\right\}$ with $c(f)>0$, where $\log _{m} x$ denotes the $m$-fold iterated logarithm. Later, a more complicated application of these ideas led P. Erdős and A. Schinzel in [2] to the conclusion that for large $x$,

$$
P_{x}>x \exp \exp \left\{c^{\prime}(f)\left(\log _{2} x\right)^{1 / 3}\right\} \quad \text { with } c^{\prime}(f)>0 .
$$

Recently, by combining Erdős' approach with an interesting result about the average order of Hooley's $\Delta$-function for polynomials, G. Tenenbaum showed in [11] that (1) can be improved to

$$
P_{x}>x \exp \left\{(\log x)^{a}\right\}
$$

for any $0<a<2-\log 4$ if $x \geq x_{0}(a, f)$.

In the following, let $K$ be a fixed algebraic number field of degree $n=$ $r_{1}+2 r_{2}$ (in the usual notation) over the rationals with discriminant $d . Z_{K}$ will denote the ring of integers in $K$.

The primary concern of the present paper is to generalize the inequality (2) to $K$ in an appropriate way. To this end, we start with an irreducible polynomial $F(x) \in Z_{K}[x]$ of degree $g>1$ and replace the integers $l \in[1, x]$ by algebraic integers $\alpha \in Z_{K}$ lying in certain lattice point regions: Let $P_{1}, \ldots, P_{n}$ be sufficiently large positive real numbers satisfying $P_{k}=P_{k+r_{2}}$ 
for $k=r_{1}+1, \ldots, r_{1}+r_{2}$ and put $P:=P_{1} \ldots P_{n}$. We consider the set

$\mathfrak{R}_{c}(P)=\left\{\alpha \in Z_{K}: \alpha\right.$ totally positive, $\left.c \leq\left|\alpha^{(k)}\right| \leq P_{k}, k=1, \ldots, n\right\}$,

where $c \geq 0$ denotes a fixed constant. Let us write $\alpha \succ 0$ to indicate that $\alpha$ is totally positive. It should be remarked that our arguments also work without the restriction $\alpha \succ 0$, but it is common practice to introduce this condition. By the theory of units (cf. [9], Hilfssatz 6) there exists a totally positive unit $\eta$ of $K$ such that

$$
P^{1 / n} \ll\left|\eta^{(k)}\right| P_{k} \ll P^{1 / n}, \quad k=1, \ldots, n .
$$

We put

$$
V:=\prod_{k=1}^{r_{1}}\left(P_{k}-c\right) \prod_{k=1}^{r_{2}}\left(P_{k+r_{1}}^{2}-c^{2}\right)
$$

and obtain, for $\min _{k} P_{k} \geq 2 c$,

$$
\left|\eta^{(k)}\right|\left(P_{k}-c\right) \gg\left|\eta^{(k)}\right| P_{k} \gg P^{1 / n} \geq V^{1 / n}, \quad k=1, \ldots, n .
$$

These observations enable us to apply Hilfssatz 9 of [7], which involves the formula

$$
\begin{aligned}
& \left|\left\{\alpha \in Z_{K}: \alpha \succ 0, \alpha \equiv \gamma \bmod \mathfrak{a}, c \leq\left|\alpha^{(k)}\right| \leq P_{k}, k=1, \ldots, n\right\}\right| \\
& =\mid\left\{\beta \in Z_{K}:\right. \\
& \left.\quad \beta \succ 0, \beta \equiv \gamma \cdot \eta \bmod \mathfrak{a}, c\left|\eta^{(k)}\right| \leq\left|\beta^{(k)}\right| \leq\left|\eta^{(k)}\right| P_{k}, k=1, \ldots, n\right\} \mid \\
& =\frac{(2 \pi)^{r_{2}}}{|\sqrt{d}|} \cdot \frac{V}{N \mathfrak{a}}+O\left\{\left(\frac{V}{N \mathfrak{a}}\right)^{1-1 / n}+1\right\},
\end{aligned}
$$

where $\mathfrak{a}$ stands for an integral ideal of $K$. Here and in the sequel, $|\ldots|$ denotes the cardinality of a finite set.

Theorem 1. Let $F(x) \in Z_{K}[x]$ be an irreducible polynomial of degree $g>1$. Denote by $\mathfrak{p}_{P}$ a greatest prime ideal factor of $\prod_{\alpha \in \mathfrak{R}_{0}(P)} F(\alpha)$, greatest in the sense that its norm $N \mathfrak{p}_{P}$ is maximal. Then for any $0<a<2-\log 4$,

$$
N \mathfrak{p}_{P}>P \exp \left\{(\log P)^{a}\right\} \quad \text { if } P \geq P_{0}(a, F, K) .
$$

The proof of Theorem 1 is influenced by the cited papers of Nagell, Erdös and Tenenbaum on the corresponding problem in the rational case. It is possible to model their main arguments appropriate to our situation. Let us only explain some of the additional difficulties.

Often one is allowed to assume without loss of generality that the $P_{k}$ obey the inequalities $P^{1 / n} \ll P_{k} \ll P^{1 / n}$ for $k=1, \ldots, n$. However, this argument does not work in our case, since multiplying $\alpha \in \mathfrak{R}_{0}(P)$ by the unit $\eta$ in (3) results in another polynomial $F$ in Theorem 1, whose coefficients depend in an indeterminable manner on this unit. We avoid this problem 
by introducing an unusual "one-dimensional" condition in the definition of the set $\mathfrak{S}_{c}(P)$ in $(15)$. Moreover, in contrast to $K=\mathbb{Q}$, the possibility of choosing the constant $c$ freely is crucial for our investigations. In particular, these modifications involve some more careful calculations in comparison with the rational case.

To explain another novel feature of the present paper a certain amount of preparation is required. Let us begin by noting that, in view of (4),

$$
\sum_{\substack{\alpha \in \Re_{c}(P) \\ F(\alpha) \equiv 0 \bmod \mathfrak{a}}} 1 \gg \frac{P}{N \mathfrak{a}} \sum_{\substack{\gamma \bmod \mathfrak{a} \\ F(\gamma) \equiv 0 \bmod \mathfrak{a}}} 1
$$

if $N \mathfrak{a} \leq c_{0} P$, where $c_{0}=c_{0}(K)$ has to be sufficiently small. The constant $c_{0}$ does not occur in the rational case.

For brevity we put $I=\left[c_{0} P / 2, c_{0} P\right]$ and denote by $D(\alpha, I)$ the number of ideal divisors $\mathfrak{a}$ of $\alpha \in Z_{K}$ satisfying $N \mathfrak{a} \in I$. Suitable extensions of some results of [1] and [2] lead us to

$$
N \mathfrak{p}_{P}>P \exp \left\{\frac{|\sqrt{d}|}{(2 \pi)^{r_{2}}} \cdot \frac{1}{g} \cdot \frac{\log P}{V} \cdot N(P)\right\}
$$

if $P \log _{2} P=o(N(P) \log P)$ as $P \rightarrow \infty$, where $N(P)$ is defined by

$$
N(P)=\left|\left\{\alpha \in \mathfrak{R}_{c}(P): D(F(\alpha), I) \geq 1\right\}\right| .
$$

Consequently, our problem has been reduced to that of establishing a suitable lower bound for $N(P)$. To this end, we apply a method which is an extension of the one developed by Tenenbaum in [11]. A key step, which is of some interest in itself, is the determination of an upper bound for the sum

$$
\sum_{\alpha \in \mathfrak{R}_{0}(P)} \Delta(F(\alpha))^{t}, \quad t \geq 1,
$$

where in our terminology Hooley's $\Delta$-function is of the form

$$
\Delta(\mathfrak{a})=\max _{u \in \mathbb{R}}|\{\mathfrak{b} \mid \mathfrak{a}: u<\log N \mathfrak{b} \leq u+1\}| .
$$

Theorem 2. For any real number $t \geq 1$ we have

$$
\sum_{\alpha \in \mathfrak{R}_{0}(P)} \Delta(F(\alpha))^{t} \ll_{t} P(\log P)^{2^{t}-t-1+o(1)}, \quad P \rightarrow \infty .
$$

It turns out that one of the main difficulties arises in connection with the transformation of the multidimensional sum (7) into the one-dimensional expression

$$
\sum_{N \mathfrak{a} \leq X} \frac{L(\mathfrak{a})}{N \mathfrak{a}} \Delta(\mathfrak{a})^{t}, \quad t \geq 1,
$$


where $L(\mathfrak{a})$ denotes the number of solutions of the congruence $F(\alpha) \equiv$ $0 \bmod \mathfrak{a}$. Using a suitable algebraic Selberg-type sieve we succeed in carrying out this reduction. The argument presented here further shows that the full rational theory developed by Tenenbaum in [10] in a more general context can be avoided. Evolving a direct proof of Theorem 2 many simplifications are possible. The procedure for the estimation of $(8)$ is now very similar to that in the rational case.

The numbers $c_{1}, \ldots, c_{26}$ coming up in the sequel are positive constants which depend at most on the field $K$ and on the coefficients and degree of $F$. Throughout, small German letters stand for integral ideals of $K$; in particular, $\mathfrak{p}$ always denotes a prime ideal.

2. Let $F(x) \in Z_{K}[x]$ be an irreducible polynomial of degree $g>1$. Denote by $\theta$ the discriminant of $F$ and by $L(\mathfrak{a})$ the number of (incongruent) solutions in $Z_{K}$ of $F(\alpha) \equiv 0 \bmod \mathfrak{a}$. We quote from [8] the relations

$$
\begin{gathered}
\sum_{N \mathfrak{p} \leq X} L(\mathfrak{p})=\operatorname{li} X+O\left\{X \exp \left(-c_{1} \sqrt{\log X}\right)\right\}, \\
\sum_{N \mathfrak{a} \leq X} L(\mathfrak{a})=c_{2} X+O\left(X^{1-b}\right), \quad 0<b \leq 1 / 2, \\
L\left(\mathfrak{p}^{l}\right) \leq \min \{g, N \mathfrak{p}-1\}, \quad \mathfrak{p} \nmid \theta_{0}:=\theta F(1), l \geq 1 .
\end{gathered}
$$

In (11) we have used the fact that $L(\mathfrak{p})<N \mathfrak{p}$ if $\mathfrak{p} \nmid F(1)$. Moreover, it is easily seen that $L\left(\mathfrak{p}^{l}\right) \ll 1$ if $\mathfrak{p} \mid \theta_{0}$.

A straightforward partial summation shows that (9) implies

$$
\sum_{N \mathfrak{p} \leq X} L(\mathfrak{p}) \frac{\log N \mathfrak{p}}{N \mathfrak{p}}=\log X+O(1)
$$

and

$$
\sum_{N \mathfrak{p} \leq X} \frac{L(\mathfrak{p})}{N \mathfrak{p}}=\log \log X+c_{3}+O\left\{\exp \left(-c_{4} \sqrt{\log X}\right)\right\},
$$

so that in particular

$$
\frac{1}{2} \log \frac{\log Y}{\log X} \leq \sum_{X<N \mathfrak{p} \leq Y} \frac{L(\mathfrak{p})}{N \mathfrak{p}} \leq 2 \log \frac{\log Y}{\log X}, \quad Y \geq 2 X \geq X_{0} .
$$

We begin our investigations by deriving the bound (6) for $N \mathfrak{p}_{P}$. To this end, let us decompose $(F(\alpha)) \neq(1)$ for $\alpha \in Z_{K}$ in the form

$$
(F(\alpha))=\mathfrak{q}_{1}(\alpha) \mathfrak{q}_{2}(\alpha),
$$

where all prime ideal factors of $\mathfrak{q}_{1}(\alpha)$ (resp. $\mathfrak{q}_{2}(\alpha)$ ) have norms $\leq Z$ (resp. $>Z$ ). The parameter $Z \geq 2$ will be chosen later. 
We introduce the set $\mathfrak{S}_{c}(P)$ of all integers $\alpha \in \mathfrak{R}_{c}(P)$ subject to the condition

$$
N \alpha>P(\log P)^{-r_{1}-r_{2}},
$$

for which $F(\alpha)$ has no prime ideal factor $\mathfrak{p}$ satisfying $c_{0} P<N \mathfrak{p} \leq c_{6} c_{0} P$, where $c_{6} \geq 1$ is chosen in such a way that $c_{7}=\frac{1}{2} c_{0}\left(c_{0} c_{6}\right)^{g-1} \geq 1$ and

$$
\frac{P^{g}}{(\log P)^{\left(r_{1}+r_{2}\right) g}} \ll|N(F(\alpha))| \leq c_{7} P^{g} \quad \text { for } \alpha \in \mathfrak{S}_{c}(P) .
$$

For sufficiently large $c=c(F, K)$ occurring in the definition of $\mathfrak{R}_{c}(P)$ this can clearly be done.

Let us pause to determine the cardinality of this set $\mathfrak{S}_{c}(P)$. Using (4) and (11) we obtain

$$
\begin{aligned}
\left|\mathfrak{S}_{c}(P)\right|= & \sum_{\alpha \in \mathfrak{R}_{c}(P)} 1+O\left\{\sum_{\substack{\alpha \in \mathfrak{R}_{0}(P) \\
N \alpha \leq P(\log P)^{-r_{1}-r_{2}}}} 1+\sum_{c_{0} P<N \mathfrak{p} \leq c_{0} c_{6} P} \sum_{\substack{\alpha \in \mathfrak{R}_{0}(P) \\
F(\alpha) \equiv 0 \bmod \mathfrak{p}}} 1\right\} \\
= & \frac{(2 \pi)^{r_{2}}}{|\sqrt{d}|} V \\
& +O\left\{P^{1-1 / n}+\sum_{\substack{\alpha \in \Re_{0}(P) \\
N \alpha \leq P(\log P)^{-r_{1}-r_{2}}}} 1+\sum_{c_{0} P<N \mathfrak{p} \leq c_{0} c_{6} P}\left(\frac{P}{N \mathfrak{p}}+1\right)\right\} \\
= & \frac{(2 \pi)^{r_{2}}}{|\sqrt{d}|} V+O\left\{\sum_{\substack{\alpha \in \Re_{0}(P) \\
N \alpha \leq(\log P)^{-r_{1}-r_{2}}}} 1+P(\log P)^{-1}\right\} .
\end{aligned}
$$

It remains to evaluate the sum occurring in the remainder term on the right. To this end we first note that

$$
\begin{aligned}
& \sum_{\substack{\alpha \in \Re_{0}(P) \\
N \alpha \leq P(\log P)^{-r_{1}-r_{2}}}} 1 \\
\leq & \left|\left\{\beta \in Z_{K},\left|\beta^{(k)}\right| \leq\left|\eta^{(k)}\right| P_{k}, k=1, \ldots, n,|N \beta| \leq P(\log P)^{-r_{1}-r_{2}}\right\}\right| \\
= & \sum_{\substack{(\beta) \\
N(\beta) \leq(\log P)^{-r_{1}-r_{2}}}} \sum_{\substack{\varepsilon(k) \\
\beta^{(k)}|\leq| \eta^{(k)} \mid P_{k}}} 1,
\end{aligned}
$$

where the outer sum is taken over all principal ideals $(\beta)$ with norms $\leq$ $P(\log P)^{-r_{1}-r_{2}}$. The inner sum gives the number $L(\beta)$ of units $\varepsilon$ of $K$ such that $\left|\varepsilon^{(k)} \beta^{(k)}\right| \leq\left|\eta^{(k)}\right| P_{k}$ for $k=1, \ldots, n$. By choosing a fixed generator $\beta$ of $(\beta)$ satisfying $|N \beta|^{1 / n} \ll\left|\beta^{(k)}\right| \ll|N \beta|^{1 / n}, k=1, \ldots, n$, it follows from (3) that 


$$
\begin{aligned}
\left(\frac{P}{|N \beta|}\right)^{1 / n-1} & \ll \prod_{j \neq k}\left|\varepsilon^{(j)}\right|^{-1}=\left|\varepsilon^{(k)}\right| \\
& \leq \frac{\left|\eta^{(k)}\right| P_{k}}{\left|\beta^{(k)}\right|} \ll\left(\frac{P}{|N \beta|}\right)^{1 / n}, \quad k=1, \ldots, n .
\end{aligned}
$$

Let $\varepsilon_{1}, \ldots, \varepsilon_{r}$ be the fundamental units of $K$ so that

$$
\left|\varepsilon^{(k)}\right|=\left|\varepsilon_{1}^{(k) l_{1}} \ldots \varepsilon_{r}^{(k) l_{r}}\right|, \quad l_{j} \in \mathbb{Z}, j=1, \ldots, r:=r_{1}+r_{2}-1 .
$$

This leads us immediately to the inequalities

$$
\left|\sum_{j=1}^{r} l_{j} \log \right| \varepsilon_{j}^{(k)}|| \ll \log \frac{P}{|N \beta|}, \quad k=1, \ldots, n .
$$

Since the rank of the matrix $A=\left(\log \left|\varepsilon_{j}^{(k)}\right|\right)^{(r, n)}$ is equal to $r$, there exists a matrix $B=\left(b_{j k}\right)^{(n, r)}$ such that the product $A B$ is the unit matrix. Hence

$$
\left|l_{q}\right|=\left|\sum_{k=1}^{n} b_{k q} \sum_{j=1}^{r} l_{j} \log \right| \varepsilon_{j}^{(k)}|| \ll \log \frac{P}{|N \beta|}, \quad q=1, \ldots, r
$$

and therefore

$$
L(\beta) \ll\left(\log \frac{P}{|N \beta|}\right)^{r_{1}+r_{2}-1} .
$$

It should be noted that the contribution of the roots of unity here gives nothing important. Returning to our starting problem we obtain

$$
\sum_{\substack{\alpha \in \Re_{0}(P) \\ \alpha \leq P(\log P)^{-r_{1}-r_{2}}}} 1 \ll(\log P)^{r_{1}+r_{2}-1} \sum_{N \mathfrak{a} \leq P(\log P)^{-r_{1}-r_{2}}} 1 \ll P(\log P)^{-1}
$$

so that finally

$$
\left|\mathfrak{S}_{c}(P)\right|=\frac{(2 \pi)^{r_{2}}}{|\sqrt{d}|} V+O\left\{P(\log P)^{-1}\right\} .
$$

The key idea is now to consider the expression $\sum_{\alpha \in \mathfrak{R}_{c}(P)} \log N \mathfrak{q}_{1}(\alpha)$. A fairly simple argument is sufficient to derive an upper bound for this sum. Starting from the inequality

$$
\sum_{\alpha \in \mathfrak{R}_{c}(P)} \log N \mathfrak{q}_{1}(\alpha) \leq \sum_{N \mathfrak{p} \leq Z} \log N \mathfrak{p} \sum_{m=1}^{M(P, \mathfrak{p})} \sum_{\substack{\alpha \in \mathfrak{R}_{c}(P) \\ F(\alpha) \equiv 0 \bmod \mathfrak{p}^{m}}} 1,
$$

where

$$
M(P, \mathfrak{p})=\max _{\alpha \in \mathfrak{R}_{c}(P)} \max _{\mathfrak{p}^{m} \mid F(\alpha)} m \ll \frac{\log P}{\log N \mathfrak{p}}+1,
$$


we have, in view of (4), (11) and (12),

$$
\begin{aligned}
& \sum_{\alpha \in \mathfrak{R}_{c}(P)} \log N \mathfrak{q}_{1}(\alpha) \\
& \leq \sum_{N \mathfrak{p} \leq Z} \log N \mathfrak{p} \sum_{\substack{\gamma \bmod \mathfrak{p} \\
F(\gamma) \equiv 0 \bmod \mathfrak{p}}} \sum_{\substack{\alpha \in \gamma \bmod \mathfrak{p} \\
\alpha \equiv \mathfrak{R}_{c}(P)}} 1 \\
& \quad+O\left\{\sum_{N \mathfrak{p} \leq Z} \log N \mathfrak{p} \sum_{m=2}^{M(P, \mathfrak{p})}\left(\frac{P}{N \mathfrak{p}^{m}}+1\right)\right\} \\
& =\frac{(2 \pi)^{r_{2}}}{|\sqrt{d}|} V \sum_{N \mathfrak{p} \leq Z} L(\mathfrak{p}) \frac{\log N \mathfrak{p}}{N \mathfrak{p}}+O\left\{P^{1-1 / n} \sum_{N \mathfrak{p} \leq Z} \frac{\log N \mathfrak{p}}{N \mathfrak{p}^{1-1 / n}}\right\} \\
& \quad+O\left\{Z+P \sum_{N \mathfrak{p} \leq Z} \frac{\log N \mathfrak{p}}{N \mathfrak{p}^{2}}+Z \frac{\log P}{\log Z}\right\} \\
& =\frac{(2 \pi)^{r_{2}}}{|\sqrt{d}|} V \log Z+O\left\{P^{1-1 / n} Z^{1 / n}+Z+P+Z \frac{\log P}{\log Z}\right\}
\end{aligned}
$$

The comparison of the upper bound (17) with a lower bound will yield the stated estimate for $N \mathfrak{p}_{P}$ in (6). The proof requires, however, some preparation.

Lemma 1. If $\alpha \in \mathfrak{S}_{c}(P)$ and $Z=c_{0} P$ in (14), then

$$
N \mathfrak{q}_{1}(\alpha) \geq \frac{c_{8} P^{g}}{(\log P)^{\left(r_{1}+r_{2}\right) g} N \mathfrak{p}_{P}^{g-1}} .
$$

Proof. If $Z=c_{0} P$, then all prime ideal factors $\mathfrak{p}$ of $\mathfrak{q}_{2}(\alpha)$ in (14) satisfy $N \mathfrak{p}>c_{6} c_{0} P$ for $\alpha \in \mathfrak{S}_{c}(P)$, so that, in view of our choice of $c_{6}, \mathfrak{q}_{2}(\alpha)$ can have at most $g-1$ prime ideal divisors, counted according to multiplicity. From this fact and (15) we deduce readily

$$
N \mathfrak{q}_{1}(\alpha)=\frac{|N(F(\alpha))|}{N \mathfrak{q}_{2}(\alpha)} \geq \frac{c_{8} P^{g}}{(\log P)^{\left(r_{1}+r_{2}\right) g} N \mathfrak{p}_{P}^{g-1}} .
$$

Lemma 2. Choose $Z=c_{0} P$ in (14) and let $\alpha \in \mathfrak{S}_{c}(P)$ satisfy $D(F(\alpha), I)$ $\geq 1$, then

$$
N \mathfrak{q}_{1}(\alpha) \geq \frac{c_{8} P^{g}}{(\log P)^{\left(r_{1}+r_{2}\right) g} N \mathfrak{p}_{P}^{g-2}} .
$$

Proof. Since there exists an ideal factor $\mathfrak{a}$ of $F(\alpha)$ with $c_{0} P / 2 \leq N \mathfrak{a} \leq$ $c_{0} P$ we have by (15) and our choice of $c_{6}$,

$$
N \mathfrak{q}_{2}(\alpha)=\frac{|N(F(\alpha))|}{N \mathfrak{q}_{1}(\alpha)} \leq \frac{2 c_{7}}{c_{0}} P^{g-1}=\left(c_{0} c_{6} P\right)^{g-1} .
$$


On the other hand, for $\alpha \in \mathfrak{S}_{c}(P)$, all prime ideal divisors $\mathfrak{p}$ of $\mathfrak{q}_{2}(\alpha)$ satisfy $N \mathfrak{p}>c_{0} c_{6} P$, so that $\mathfrak{q}_{2}(\alpha)$ can have at most $g-2$ prime ideal factors, multiple factors counted multiple. Hence, by (15),

$$
N \mathfrak{q}_{1}(\alpha)=\frac{|N(F(\alpha))|}{N \mathfrak{q}_{2}(\alpha)} \geq \frac{c_{8} P^{g}}{(\log P)^{\left(r_{1}+r_{2}\right) g} N \mathfrak{p}_{P}^{g-2}} .
$$

We are now in a position to derive a suitable lower bound for the sum under consideration. An appeal to Lemmas 1 and 2 leads us at once to

$$
\begin{aligned}
& \sum_{\alpha \in \mathfrak{S}_{c}(P)} \log N \mathfrak{q}_{1}(\alpha) \\
& \geq\left(g \log P-\left(r_{1}+r_{2}\right) g \log _{2} P-(g-2) \log N \mathfrak{p}_{P}+\log c_{8}\right) \sum_{\substack{\alpha \in \mathfrak{S}_{c}(P) \\
D(F(\alpha), I) \geq 1}} 1 \\
& +\left(g \log P-\left(r_{1}+r_{2}\right) g \log _{2} P-(g-1) \log N \mathfrak{p}_{P}+\log c_{8}\right) \sum_{\substack{\alpha \in \mathfrak{S}_{c}(P) \\
D(F(\alpha), I)=0}} 1 \\
& =\log N \mathfrak{p}_{P} \cdot \sum_{\substack{\alpha \in \mathfrak{S}_{c}(P) \\
D(F(\alpha), I) \geq 1}} 1 \\
& +\left(g \log P-\left(r_{1}+r_{2}\right) g \log _{2} P-(g-1) \log N \mathfrak{p}_{P}+\log c_{8}\right) \sum_{\alpha \in \mathfrak{S}_{c}(P)} 1 .
\end{aligned}
$$

We further note the inequality

$$
\sum_{\substack{\alpha \in \mathfrak{S}_{c}(P) \\ D(F(\alpha), I) \geq 1}} 1 \geq N(P)-\left\{\sum_{\alpha \in \mathfrak{R}_{c}(P)} 1-\sum_{\alpha \in \mathfrak{S}_{c}(P)} 1\right\},
$$

so that finally by (4), (15) and (16),

$$
\begin{aligned}
& \sum_{\alpha \in \mathfrak{S}_{c}(P)} \log N \mathfrak{q}_{1}(\alpha) \\
& \geq \log N \mathfrak{p}_{P} \cdot\left\{N(P)-c_{9} P(\log P)^{-1}\right\} \\
&+\left(g \log P-\left(r_{1}+r_{2}\right) g \log _{2} P-(g-1) \log N \mathfrak{p}_{P}+\log c_{8}\right) \\
& \times\left(\frac{(2 \pi)^{r_{2}}}{|\sqrt{d}|} V-c_{10} P(\log P)^{-1}\right) \\
& \geq \frac{(2 \pi)^{r_{2}}}{|\sqrt{d}|}\left(g V \log P-\left(r_{1}+r_{2}\right) g P \log _{2} P-(g-1) V \log N \mathfrak{p}_{P}\right) \\
&+N(P) \log N \mathfrak{p}_{P}-c_{11} P .
\end{aligned}
$$


If we combine (18) with (17) for $Z=c_{0} P$, we arrive at

$$
\frac{(2 \pi)^{r_{2}}}{|\sqrt{d}|}(g-1) V \log \frac{N \mathfrak{p}_{P}}{P} \geq N(P) \log N \mathfrak{p}_{P}-c_{12} P \log _{2} P-c_{13} P .
$$

Since, for sufficiently large $P,(13)$ implies the existence of a prime ideal $\mathfrak{p}$ satisfying $c_{0} P / 2<N \mathfrak{p} \leq c_{0} P$ and $L(\mathfrak{p})>0$, we deduce at once from (5) that

$$
N \mathfrak{p}_{P}>c_{0} P / 2 \quad \text { for } P \geq P_{0}(F, K) .
$$

Let us now assume that $P \log _{2} P=o(N(P) \log P)$ as $P \rightarrow \infty$. Then we have, for sufficiently large $P$,

$$
c_{12} P \log _{2} P+c_{13} P<\frac{1}{g} N(P) \log P+N(P) \log \frac{c_{0}}{2} .
$$

By (19) and (20), this leads us to

$$
\frac{(2 \pi)^{r_{2}}}{|\sqrt{d}|}(g-1) V \log \frac{N \mathfrak{p}_{P}}{P}>\frac{g-1}{g} N(P) \log P,
$$

so that

$$
N \mathfrak{p}_{P}>P \exp \left\{\frac{|\sqrt{d}|}{(2 \pi)^{r_{2}}} \cdot \frac{1}{g} \cdot N(P) \cdot \frac{\log P}{V}\right\}
$$

which completes the proof of $(6)$.

It remains to deal with $N(P)$. These investigations are longer and constitute the main problem to be overcome. We begin by observing that certainly

$$
N(P)=\sum_{\substack{\alpha \in \Re_{c}(P) \\ D(F(\alpha), I) \geq 1}} 1 \geq(\log P)^{-b} \sum_{\substack{\alpha \in \mathfrak{R}_{c}(P) \\ D(F(\alpha), I) \leq(\log P)^{b}}} D(F(\alpha), I),
$$

where $b>0$ will be chosen later. Recalling the definiton

$$
D(\alpha, I)=\left|\left\{\mathfrak{a} \mid \alpha: c_{0} P / 2 \leq N \mathfrak{a} \leq c_{0} P\right\}\right|
$$

already given in the introduction, an application of (5) and (10) leads us to

so that

$$
\sum_{\alpha \in \mathfrak{R}_{c}(P)} D(F(\alpha), I) \gg \sum_{c_{0} P / 2 \leq N \mathfrak{a} \leq c_{0} P} L(\mathfrak{a}) \gg P,
$$

$$
N(P) \gg P(\log P)^{-b}-(\log P)^{-b} \sum_{\substack{\alpha \in \Re_{c}(P) \\ D(F(\alpha), I)>(\log P)^{b}}} D(F(\alpha), I) .
$$

It remains to establish an upper bound for the sum

$$
\sum_{\substack{\alpha \in \mathfrak{R}_{c}(P) \\ D(F(\alpha), I)>(\log P)^{b}}} D(F(\alpha), I) \leq \sum_{\substack{\alpha \in \mathfrak{R}_{c}(P) \\ \Delta(F(\alpha))>(\log P)^{b}}} \Delta(F(\alpha)) .
$$


For $s>0$, we have

$$
\sum_{\substack{\alpha \in \Re_{c}(P) \\ \Delta(F(\alpha))>(\log P)^{b}}} \Delta(F(\alpha)) \leq(\log P)^{-b s} \sum_{\alpha \in \Re_{0}(P)} \Delta(F(\alpha))^{1+s},
$$

so that our problem has been reduced to that of proving Theorem 2. To this end, a certain amount of preparation is required. The results below form the basis of the method. We start by extending to number fields a well-known theorem due to van der Corput.

Lemma 3. For any positive real number $r$ we have

$$
\sum_{\alpha \in \Re_{0}(P)}\left\{\sum_{\mathfrak{a} \mid F(\alpha)} 1\right\}^{r} \ll_{r} P(\log P)^{2^{g r}} .
$$

Proof. We generalize to $K$ an argument of B. Landreau [5], who gives in the rational case a simple proof of van der Corput's result. The method starts from the following inequality, which holds for any positive integer $j$ :

$$
\left(\sum_{\mathfrak{a} \mid \mathfrak{b}}\right)^{r} \leq j^{j(j-1) r} \sum_{\substack{\mathfrak{a} \mid \mathfrak{b} \\ N \mathfrak{a} \leq(N \mathfrak{b})^{1 / j}}}\left(\sum_{\mathfrak{c} \mid \mathfrak{a}}\right)^{j r} .
$$

In order to justify this inequality, the procedure is in all ways analogous to that at the corresponding stage of [5]. We therefore omit the proof.

Using (15) and (24), we obtain at once

$$
\sum_{\alpha \in \Re_{0}(P)}\left\{\sum_{\mathfrak{a} \mid F(\alpha)} 1\right\}^{r} \leq j^{j(j-1) r} \sum_{N \mathfrak{a} \ll P^{g / j}}\left(\sum_{\mathfrak{c} \mid \mathfrak{a}} 1\right)^{j r} \sum_{\substack{\alpha \in \Re_{0}(P) \\ F(\alpha) \equiv 0 \bmod \mathfrak{a}}} 1 .
$$

If we choose $j=g$ and take (4) into account, the sum under consideration is of order

$$
\ll_{r} P \sum_{N \mathfrak{a} \ll P} D(\mathfrak{a})^{g r} \frac{L(\mathfrak{a})}{N \mathfrak{a}},
$$

where $D(\mathfrak{a})$ denotes the number of ideal divisors of $\mathfrak{a}$. It remains to deal with the sum

$$
\sum_{N \mathfrak{a} \leq X} D(\mathfrak{a})^{m} \frac{L(\mathfrak{a})}{N \mathfrak{a}}, \quad m \in \mathbb{N} .
$$

Here, we proceed by induction on $m$. Let us only consider the case $m=1$. An appeal to (10) shows that

$$
\sum_{N \mathfrak{a} \leq X} D(\mathfrak{a}) \frac{L(\mathfrak{a})}{N \mathfrak{a}} \leq \sum_{N \mathfrak{b} \leq X} \frac{L(\mathfrak{b})}{N \mathfrak{b}} \sum_{N \mathfrak{a} \leq X / N \mathfrak{b}} \frac{L(\mathfrak{a})}{N \mathfrak{a}} \ll \log ^{2} X .
$$


In general we obtain

$$
\sum_{N \mathfrak{a} \leq X} D(\mathfrak{a})^{m} \frac{L(\mathfrak{a})}{N \mathfrak{a}} \ll(\log X)^{2^{m}} .
$$

This completes the proof of Lemma 3.

The second main tool in the proof of Theorem 2 is the following result which is based on a sieve technique.

$$
\begin{aligned}
& \text { Lemma 4. If } Y \geq Y_{0}(F, K) \geq 2 \text {, then } \\
& \begin{aligned}
\mid\left\{\alpha \in \mathfrak{R}_{0}(P): F(\alpha) \equiv\right. & \left.0 \bmod \mathfrak{a}, \mathfrak{p} \mid F(\alpha) \Rightarrow N \mathfrak{p}>Y \text { or } \mathfrak{p} \mid \mathfrak{a} \theta_{0}\right\} \mid \\
\leq & \frac{w}{2^{r_{1}} h R} \cdot \frac{N\left(\theta_{0}\right)}{\phi\left(\theta_{0}\right)} \prod_{\mathfrak{p} \nmid \theta_{0}} \frac{1-L(\mathfrak{p}) / N \mathfrak{p}}{1-1 / N \mathfrak{p}} \\
& \times \prod_{\mathfrak{p} \mid \mathfrak{a}, \mathfrak{p} \nmid \theta_{0}}\left(1-\frac{L(\mathfrak{p})}{N \mathfrak{p}}\right)^{-1} \frac{L(\mathfrak{a})}{N \mathfrak{a}} \cdot \frac{P}{\log Y} \\
& \times\left\{1+O\left(\frac{\log \log 3 N \mathfrak{a}}{\log Y}\right)\right\} \\
& +O\left\{L(\mathfrak{a})\left(\frac{P}{N \mathfrak{a}}\right)^{1-1 / n} Y^{2 / n} \log ^{3} Y+L(\mathfrak{a}) Y^{2}\right\},
\end{aligned}
\end{aligned}
$$

where $w$ denotes the number of roots of unity, $h$ the class number and $R$ the regulator of the field $K$.

Proof. We make use of a Selberg-type upper bound sieve in $K$, as described in [8] (see also [4]). Let us take as the sequence to be sifted

$$
\mathfrak{A}=\left\{F(\alpha): \alpha \in \mathfrak{R}_{0}(P), F(\alpha) \equiv 0 \bmod \mathfrak{a}\right\},
$$

and as the sifting set

$$
\mathfrak{P}=\left\{\mathfrak{p} \subset Z_{K}: N \mathfrak{p} \leq Y, \mathfrak{p} \nmid \mathfrak{a} \theta_{0}\right\} .
$$

Using the abbreviation

$$
T(Y)=\prod_{\substack{\mathfrak{p} \in \mathfrak{P} \\ L(\mathfrak{p}) \neq 0}} \mathfrak{p}
$$

we see at once that

$$
\sum_{\substack{\alpha \in \mathfrak{R}_{0}(P) \\
F(\alpha) \equiv 0 \bmod \mathfrak{a} \\
(\alpha) \Rightarrow N \mathfrak{p}>Y \text { or } \mathfrak{p} \mid \mathfrak{a} \theta_{0}}} 1 \leq \sum_{\begin{array}{c}
\alpha \in \mathfrak{R}_{0}(P) \\
F(\alpha) \equiv 0 \bmod \mathfrak{a} \\
(F(\alpha), T(Y))=1
\end{array}} 1 .
$$

The expression on the right of (25) has already been considered in [8]. There, 
on page 450 , we find the estimate

$$
\begin{aligned}
& \sum_{\substack{\alpha \in \mathfrak{R}_{0}(P) \\
F(\alpha)=0 \bmod \mathfrak{a} \\
(F(\alpha), T(Y))=1}} 1 \leq \frac{(2 \pi)^{r_{2}}}{|\sqrt{d}|} P \frac{L(\mathfrak{a})}{N \mathfrak{a}} S^{-1}(\mathfrak{a}, Y) \\
& +O\left\{L(\mathfrak{a})\left(\frac{P}{N \mathfrak{a}}\right)^{1-1 / n} Y^{2 / n} \log ^{3} Y+L(\mathfrak{a}) Y^{2}\right\},
\end{aligned}
$$

where

$$
\begin{aligned}
S(\mathfrak{a}, Y) & =\sum_{\substack{N \mathfrak{b} \leq Y \\
\mathfrak{b} \mid T(Y)}} \frac{L(\mathfrak{b})}{N \mathfrak{b}} \prod_{\mathfrak{p} \mid \mathfrak{b}}\left(1-\frac{L(\mathfrak{p})}{N \mathfrak{p}}\right)^{-1} \\
& =\sum_{\substack{N \mathfrak{b} \leq Y \\
\left(\mathfrak{b}, \mathfrak{a} \theta_{0}\right)=1}} \mu(\mathfrak{b})^{2} \frac{L(\mathfrak{b})}{N \mathfrak{b}} \prod_{\mathfrak{p} \mid \mathfrak{b}}\left(1-\frac{L(\mathfrak{p})}{N \mathfrak{p}}\right)^{-1} .
\end{aligned}
$$

The problem is now to investigate the asymptotic behaviour of the function $S(\mathfrak{a}, Y)$. Arguing in precisely the same way as in Lemma 2.5 of [4] we obtain

$$
\begin{aligned}
S^{-1}(\mathfrak{a}, Y)= & e^{\gamma_{0}} \prod_{\mathfrak{p} \in \mathfrak{p}}\left(1-\frac{L(\mathfrak{p})}{N \mathfrak{p}}\right)\left\{1+O\left(\frac{\log \log 3 N \mathfrak{a}}{\log Y}\right)\right\} \\
= & e^{\gamma_{0}} \prod_{\substack{N \mathfrak{p} \leq Y \\
\mathfrak{p} \mid \mathfrak{a}, \mathfrak{p} \nmid \theta_{0}}}\left(1-\frac{L(\mathfrak{p})}{N \mathfrak{p}}\right)^{-1} \prod_{\substack{N \mathfrak{p} \leq Y \\
\mathfrak{p} \nmid \theta_{0}}}\left(1-\frac{L(\mathfrak{p})}{N \mathfrak{p}}\right) \\
& \times\left\{1+O\left(\frac{\log \log 3 N \mathfrak{a}}{\log Y}\right)\right\} .
\end{aligned}
$$

A simple calculation shows that

$$
\prod_{\substack{N \mathfrak{p}>Y \\ \mathfrak{p} \mid \mathfrak{a}, \mathfrak{p} \nmid \theta_{0}}}\left(1-\frac{L(\mathfrak{p})}{N \mathfrak{p}}\right)=1+O\left(\frac{\log \log 3 N \mathfrak{a}}{\log Y}\right) .
$$

This relation is trivial if $\log 3 N \mathfrak{a} \geq Y$; otherwise we have by (11),

$$
\begin{aligned}
1 & \geq \prod_{\substack{N \mathfrak{p}>Y \\
\mathfrak{p} \mid \mathfrak{a}, \mathfrak{p} \nmid \theta_{0}}}\left(1-\frac{L(\mathfrak{p})}{N \mathfrak{p}}\right) \geq\left(1-\frac{g}{Y}\right)^{v(\mathfrak{a})} \geq 1-\frac{g v(\mathfrak{a})}{Y} \\
& \geq 1-c_{14} \frac{\log N \mathfrak{a}}{Y \log \log 3 N \mathfrak{a}} \geq 1-\frac{c_{15}}{\log Y},
\end{aligned}
$$

where $v(\mathfrak{a}) \ll N \mathfrak{a} / \log \log 3 N \mathfrak{a}$ denotes the number of distinct prime ideal divisors of $\mathfrak{a}$. 
To complete the proof of Lemma 4 it only remains to apply the formula (see e.g. [8], Lemma 4.1)

$$
\begin{aligned}
\prod_{\substack{N \mathfrak{p} \leq Y \\
\mathfrak{p} \nmid \theta_{0}}}\left(1-\frac{L(\mathfrak{p})}{N \mathfrak{p}}\right)= & e^{-\gamma_{0}} \frac{w|\sqrt{d}|}{(2 \pi)^{r_{2}} 2^{r_{1}} h R} \prod_{\mathfrak{p} \nmid \theta_{0}} \frac{1-L(\mathfrak{p}) / N \mathfrak{p}}{1-1 / N \mathfrak{p}} \prod_{\mathfrak{p} \mid \theta_{0}}\left(1-\frac{1}{N \mathfrak{p}}\right)^{-1} \\
& \times \frac{1}{\log Y}\left\{1+O\left(\frac{1}{\log Y}\right)\right\} .
\end{aligned}
$$

Finally, in the proof of Theorem 2 we shall make use of the following result.

Lemma 5. Let $t \geq 1$ be a real number. Then

$$
\sum_{N \mathfrak{a} \leq X} \frac{L(\mathfrak{a})}{N \mathfrak{a}} \Delta(\mathfrak{a})^{t} \ll_{t}(\log X)^{2^{t}-t+o(1)} \quad \text { as } X \rightarrow \infty .
$$

P r o of. We argue in the same way as Tenenbaum did in [11], Lemma 2.2. For the sake of completeness let us repeat the main steps.

We start by recalling from the introduction the definition

$$
\Delta(\mathfrak{a})=\max _{u \in \mathbb{R}} \Delta(\mathfrak{a}, u),
$$

where

$$
\Delta(\mathfrak{a}, u)=|\{\mathfrak{b} \mid \mathfrak{a}: u<\log N \mathfrak{b} \leq u+1\}| .
$$

For an integer $q \geq 2$, consider

$$
M_{q}(\mathfrak{a})=\int_{-\infty}^{\infty} \Delta(\mathfrak{a}, u)^{q} d u
$$

It is easily seen that

$$
2^{1-q} \Delta(\mathfrak{a})^{q} \leq M_{q}(\mathfrak{a}) \leq D(\mathfrak{a})^{q},
$$

where again $D(\mathfrak{a})$ denotes the number of ideal divisors of $\mathfrak{a}$. Let us now introduce the series

$$
L(\sigma)=\sum_{\mathfrak{a}} \mu(\mathfrak{a})^{2} M_{q}(\mathfrak{a})^{t / q} L(\mathfrak{a})(N \mathfrak{a})^{-\sigma}, \quad 1<\sigma \leq 2 .
$$

Making use of (26) and the simple fact that

$$
\Delta(\mathfrak{a} \mathfrak{b}) \leq \Delta(\mathfrak{a}) D(\mathfrak{b}),
$$

we obtain at once

$$
\begin{aligned}
\sum_{N \mathfrak{a} \leq X} \frac{L(\mathfrak{a})}{N \mathfrak{a}} \Delta(\mathfrak{a})^{t} & \leq \sum_{N \mathfrak{a} \leq X} \mu(\mathfrak{a})^{2} \frac{\Delta(\mathfrak{a})^{t} L(\mathfrak{a})}{N \mathfrak{a}} \sum_{\substack{\mathfrak{b} \\
\mathfrak{p}\left|\mathfrak{b} \Rightarrow \mathfrak{p}^{2}\right| \mathfrak{b}}} \frac{D(\mathfrak{b})^{t} L(\mathfrak{b})}{N \mathfrak{b}} \\
& \ll_{t} L\left(1+\frac{1}{\log X}\right) .
\end{aligned}
$$


It remains to deal with the function $L(\sigma)$. To this end we apply the method of differential inequalities as described in [3], Chapter 7. The method starts from the following identity, valid for integral ideals $\mathfrak{a}$ and prime ideals $\mathfrak{p}$ not dividing $\mathfrak{a}$ :

$$
\Delta(\mathfrak{p a}, u)=\Delta(\mathfrak{a}, u)+\Delta(\mathfrak{a}, u-\log N \mathfrak{p}) .
$$

Raising to the power $q$ and integrating, we deduce that

$$
\begin{aligned}
M_{q}(\mathfrak{p a}) & =\sum_{j=0}^{q}\left(\begin{array}{l}
q \\
j
\end{array}\right) \int_{-\infty}^{\infty} \Delta(\mathfrak{a}, u-\log N \mathfrak{p})^{j} \Delta(\mathfrak{a}, u)^{q-j} d u \\
& =2 M_{q}(\mathfrak{a})+E_{q}(\mathfrak{a}, \mathfrak{p}),
\end{aligned}
$$

where

$$
E_{q}(\mathfrak{a}, \mathfrak{p})=\sum_{j=1}^{q-1}\left(\begin{array}{l}
q \\
j
\end{array}\right) \int_{-\infty}^{\infty} \Delta(\mathfrak{a}, u-\log N \mathfrak{p})^{j} \Delta(\mathfrak{a}, u)^{q-j} d u .
$$

Let us pause to study the expression $E_{q}(\mathfrak{a}, \mathfrak{p})$. For $1 \leq j \leq q-1$, we consider

$$
\sum_{\mathfrak{p}} \Delta(\mathfrak{a}, u-\log N \mathfrak{p})^{j} \frac{\log N \mathfrak{p}}{N \mathfrak{p}},
$$

and we find immediately that an upper bound for this sum is

$$
c_{16} \sum_{\mathfrak{b}_{1} \mid \mathfrak{a}}^{\prime} \cdots \sum_{\mathfrak{b}_{j} \mid \mathfrak{a}}^{\prime} 1,
$$

where the dash means the additional summation condition

$$
\log \left(\max _{m=1, \ldots, j} N \mathfrak{b}_{m}\right)-\log \left(\min _{m=1, \ldots, j} N \mathfrak{b}_{m}\right)<1 .
$$

Hence the sum in (28) does not exceed

$$
\begin{aligned}
c_{16} \sum_{\mathfrak{b}_{1} \mid \mathfrak{a}} \ldots \sum_{\mathfrak{b}_{j} \mid \mathfrak{a}}\left(2-\log \frac{\max _{m} N \mathfrak{b}_{m}}{\min _{m} N \mathfrak{b}_{m}}\right)^{j} & =c_{16} \int_{-\infty}^{\infty}(\Delta(\mathfrak{a}, u-1)+\Delta(\mathfrak{a}, u))^{j} d u \\
& \leq c_{16} 2^{j} \int_{-\infty}^{\infty} \Delta(\mathfrak{a}, u)^{j} d u .
\end{aligned}
$$

This leads us to

$$
\sum_{\mathfrak{p}} E_{q}(\mathfrak{a}, \mathfrak{p}) \frac{\log N \mathfrak{p}}{N \mathfrak{p}} \leq c_{16} \sum_{j=1}^{q-1}\left(\begin{array}{c}
q \\
j
\end{array}\right) 2^{j} \int_{-\infty}^{\infty} \Delta(\mathfrak{a}, u)^{j} d u \int_{-\infty}^{\infty} \Delta(\mathfrak{a}, u)^{q-j} d u .
$$

Now Hölder's inequality gives, for $1 \leq m \leq q-1$, 


$$
\begin{aligned}
& \int_{-\infty}^{\infty} \Delta(\mathfrak{a}, u)^{m} d u \\
& \leq\left\{\int_{-\infty}^{\infty} \Delta(\mathfrak{a}, u) d u\right\}^{(q-m) /(q-1)}\left\{\int_{-\infty}^{\infty} \Delta(\mathfrak{a}, u)^{q} d u\right\}^{(m-1) /(q-1)},
\end{aligned}
$$

so that

$$
\begin{aligned}
& \sum_{\mathfrak{p}} E_{q}(\mathfrak{a}, \mathfrak{p}) \frac{\log N \mathfrak{p}}{N \mathfrak{p}} \\
& \quad \leq c_{16} \sum_{j=1}^{q-1}\left(\begin{array}{l}
q \\
j
\end{array}\right) 2^{j}\left\{\int_{-\infty}^{\infty} \Delta(\mathfrak{a}, u) d u\right\}^{q /(q-1)}\left\{\int_{-\infty}^{\infty} \Delta(\mathfrak{a}, u)^{q} d u\right\}^{(q-2) /(q-1)} \\
& \quad=c_{16} 4^{q} D(\mathfrak{a})^{q /(q-1)} M_{q}(\mathfrak{a})^{(q-2) /(q-1)}
\end{aligned}
$$

This completes our treatment of $E_{q}(\mathfrak{a}, \mathfrak{p})$.

Returning to (27) we obtain for $1<\sigma \leq 2$,

$$
\begin{aligned}
\sum_{\mathfrak{p}} M_{q}(\mathfrak{a}, \mathfrak{p}) & \frac{L(\mathfrak{p}) \log N \mathfrak{p}}{N \mathfrak{p}} \\
& \leq 2 M_{q}(\mathfrak{a}) \sum_{\mathfrak{p}} \frac{L(\mathfrak{p}) \log N \mathfrak{p}}{N \mathfrak{p}^{\sigma}}+c_{17} 4^{q} D(\mathfrak{a})^{q /(q-1)} M_{q}(\mathfrak{a})^{(q-2) /(q-1)} \\
& \leq 2 M_{q}(\mathfrak{a})\left\{\frac{1}{\sigma-1}+c_{18}\right\}+c_{17} 4^{q} D(\mathfrak{a})^{q /(q-1)} M_{q}(\mathfrak{a})^{(q-2) /(q-1)}
\end{aligned}
$$

on using (9) in the last step.

An application of the inequalities of Hölder and Minkowski now leads us to

$$
\begin{aligned}
-L^{\prime}(\sigma)= & \sum_{\mathfrak{a}} \mu(\mathfrak{a})^{2} M_{q}(\mathfrak{a})^{t / q} \frac{L(\mathfrak{a})}{N \mathfrak{a}^{\sigma}} \sum_{\mathfrak{p} \mid \mathfrak{a}} \log N \mathfrak{p} \\
= & \sum_{\mathfrak{p}} \sum_{\substack{\mathfrak{a} \nmid \mathfrak{a} \\
\mathfrak{p} \mathfrak{a}}} \mu(\mathfrak{a})^{2} M_{q}(\mathfrak{p a})^{t / q} \frac{L(\mathfrak{p a}) \log N \mathfrak{p}}{(N \mathfrak{p a})^{\sigma}} \\
\leq & 2^{t / q} L(\sigma)\left(\frac{1}{\sigma-1}+c_{18}\right)+c_{19}\left(\frac{1}{\sigma-1}+c_{18}\right)^{1-t / q} \\
& \times \sum_{\mathfrak{a}} \mu(\mathfrak{a})^{2} M_{q}(\mathfrak{a})^{t(q-2) /(q(q-1))} \frac{L(\mathfrak{a}) D(\mathfrak{a})^{t /(q-1)}}{N \mathfrak{a}^{\sigma}} .
\end{aligned}
$$

Proceeding now in exactly the same way as at the corresponding stage of [11], we arrive at

$$
-L^{\prime}(\sigma) \leq 2^{t / q} L(\sigma)(\sigma-1)^{-1}+c_{20} L(\sigma)^{(q-2) /(q-1)}(\sigma-1)^{t / q-1-2^{t} /(q-1)} .
$$


This differential inequality is the heart of the matter. The corresponding differential equation has solution

$$
T(\sigma)=Q(\sigma-1)^{t-2^{t}-t / q},
$$

where

$$
Q=\left(\frac{c_{20}}{2^{t}-t+t / q-2^{t / q}}\right)^{q-1} \leq\left(c_{21} q\right)^{q} .
$$

Since $L(2) \leq T(2)$, we see by Lemma 70.2 of [3] that $L(\sigma) \leq T(\sigma)$ throughout the range $1<\sigma \leq 2$. Thus, to complete the proof of Lemma 5 it only remains to choose

$$
\sigma=1+\frac{1}{\log X}, \quad q=\left[\sqrt{\frac{2 t \log \log X}{\log \log \log X}}\right] .
$$

We are now in a position to give the

Proof of Theorem 2 . For given $t \geq 1$ let $q(t)$ be a sufficiently small positive parameter that remains to be chosen. We put

$$
Z=\exp \left\{q(t) \frac{\log P}{\log \log P}\right\}
$$

Analogously to (14), we decompose $(F(\alpha)) \neq(1)$ for $\alpha \in \mathfrak{R}_{0}(P)$ in the form

$$
(F(\alpha))=\mathfrak{q}_{1}(\alpha) \mathfrak{q}_{2}(\alpha),
$$

where all prime ideal factors of $\mathfrak{q}_{1}(\alpha)$ (resp. $\mathfrak{q}_{2}(\alpha)$ ) have norms $\leq Z$ (resp. $>Z$ ).

Let us first deal with the sum

$$
\sum_{\substack{\alpha \in \Re_{0}(P) \\ N \mathfrak{q}_{1}(\alpha)>Y}} \Delta(F(\alpha))^{t}, \quad \text { where } Y \geq Z \geq 2
$$

An application of the Cauchy-Schwarz inequality yields

$$
\sum_{\substack{\alpha \in \mathfrak{R}_{0}(P) \\ N \mathfrak{q}_{1}(\alpha)>Y}} \Delta(F(\alpha))^{t} \leq\left\{\sum_{\substack{\alpha \in \mathfrak{R}_{0}(P) \\ N \mathfrak{q}_{1}(\alpha)>Y}} 1\right\}^{1 / 2}\left\{\sum_{\alpha \in \mathfrak{R}_{0}(P)} \Delta(F(\alpha))^{2 t}\right\}^{1 / 2} .
$$

For the second term on the right of (31) an appeal to Lemma 3 shows that

$$
\sum_{\alpha \in \mathfrak{R}_{0}(P)} \Delta(F(\alpha))^{2 t} \leq \sum_{\alpha \in \mathfrak{R}_{0}(P)}\left\{\sum_{\mathfrak{a} \mid F(\alpha)} 1\right\}^{2 t} \ll_{t} P(\log P)^{4^{g t}} .
$$

It remains to estimate the first expression on the right of (31). To this end, we dissect the sum under consideration into two parts $S_{1}$ and $S_{2}$ corresponding to the additional condition $N \mathfrak{k}\left(\mathfrak{q}_{1}(\alpha)\right)<Y^{1 /(3 g)}$ or $N \mathfrak{k}\left(\mathfrak{q}_{1}(\alpha)\right) \geq$ $Y^{1 /(3 g)}$ respectively, where $\mathfrak{k}(\mathfrak{a})=\prod_{\mathfrak{p} \mid \mathfrak{a}} \mathfrak{p}$ denotes the "kernel" of the ideal $\mathfrak{a}$. 
If $N \mathfrak{q}_{1}(\alpha)>Y$ and $N \mathfrak{k}\left(\mathfrak{q}_{1}(\alpha)\right)<Y^{1 /(3 g)}$ for $\alpha \in \mathfrak{R}_{0}(P)$, then

$$
\prod_{\substack{\mathfrak{p}^{l} \| \mathfrak{q}_{1}(\alpha) \\ l \geq 3 g}} N \mathfrak{p}^{l} \geq N \mathfrak{q}_{1}(\alpha) \cdot\left(N \mathfrak{k}\left(\mathfrak{q}_{1}(\alpha)\right)\right)^{-3 g+1}>Y^{1 /(3 g)},
$$

so that, making also use of (15),

$$
S_{1} \leq \sum_{\substack{Y^{1 /(3 g)}<N \mathfrak{a} \leq c_{7} P^{g} \\ \mathfrak{p}^{l} \| \mathfrak{a} \Rightarrow l \geq 3 g}} \sum_{\substack{\alpha \in \Re_{0}(P) \\ F(\alpha) \equiv 0 \bmod \mathfrak{a}}} 1 .
$$

Here, we mean as usual by $\mathfrak{p}^{l} \| \mathfrak{a}$ that $\mathfrak{p}^{l} \mid \mathfrak{a}$, but $\mathfrak{p}^{l+1} \nmid \mathfrak{a}$. Consequently, (4) and (11) lead us, for $0<u<1$, to the estimate

$$
\begin{aligned}
S_{1} & \ll \sum_{\substack{Y^{1 /(3 g)}<N \mathfrak{a} \leq c_{7} P^{g} \\
\mathfrak{p}^{l} \| \mathfrak{a} \Rightarrow l \geq 3 g}} L(\mathfrak{a})\left(\frac{P}{N \mathfrak{a}}+1\right) \\
& \ll \sum_{\substack{N \mathfrak{a} \leq c_{7} P^{g} \\
\mathfrak{p}^{l} \| \mathfrak{a} \Rightarrow l \geq 3 g}} c_{22}^{v(\mathfrak{a})}\left\{\frac{P}{N \mathfrak{a}}\left(N \mathfrak{a} \cdot Y^{-1 /(3 g)}\right)^{u}+\left(\frac{P^{g}}{N \mathfrak{a}}\right)^{1-u}\right\},
\end{aligned}
$$

where $v(\mathfrak{a})$ denotes as before the number of distinct prime ideal divisors of a. Noting that the series

$$
\sum_{\substack{\mathfrak{a} \\ \mathfrak{p}^{l} \| \mathfrak{a} \Rightarrow l \geq 3 g}} c_{22}^{v(\mathfrak{a})} \cdot N \mathfrak{a}^{u-1}
$$

is convergent for $u<1-1 /(3 g)$, we choose for instance $u=1-1 /(2 g)$ and thus obtain at once

$$
S_{1} \ll P \cdot Y^{-\frac{1}{3 g}\left(1-\frac{1}{2 g}\right)}+P^{1 / 2} .
$$

Taking $Y=P^{1 / 3}$, we see, by (31) and (32), that the contribution of $S_{1}$ to the expression (30) is of order $\ll P^{1-1 /(18 g)}$, which is acceptable in Theorem 2 .

Let us now turn our attention to

$$
\begin{aligned}
& S_{2}=\left|\left\{\alpha \in \mathfrak{R}_{0}(P): N \mathfrak{q}_{1}(\alpha)>Y, N \mathfrak{k}\left(\mathfrak{q}_{1}(\alpha)\right) \geq Y^{1 /(3 g)}\right\}\right|, \\
& \text { where } Y=P^{1 / 3} \text {. }
\end{aligned}
$$

The estimation of this cardinality runs along the lines of the proof of Lemma 3.5 in [10]. We start from the inequality

$$
S_{2} \leq Y^{-z /(3 g)} \sum_{\alpha \in \mathfrak{R}_{0}(P)}^{\prime} N \mathfrak{k}\left(\mathfrak{q}_{1}(\alpha)\right)^{z}, \quad z=1 / \log Z,
$$

where the dash indicates that summation is over those numbers $\alpha$ only for 
which $\mathfrak{q}_{1}(\alpha) \neq(1)$. Our aim is to show that

$$
\sum_{\alpha \in \Re_{0}(P)}^{\prime} N \mathfrak{k}\left(\mathfrak{q}_{1}(\alpha)\right)^{z} \ll P .
$$

To this end it is convenient to introduce a strongly multiplicative function $H$, defined by

$$
H(\mathfrak{p})= \begin{cases}N \mathfrak{p}^{z} & \text { if } N \mathfrak{p} \leq Z \\ 1 & \text { if } N \mathfrak{p}>Z\end{cases}
$$

so that

$$
\sum_{\alpha \in \mathfrak{R}_{0}(P)}^{\prime} N \mathfrak{k}\left(\mathfrak{p}_{1}(\alpha)\right)^{z}=\sum_{\alpha \in \mathfrak{R}_{0}(P)}^{\prime} H(F(\alpha)) .
$$

For any $\alpha \in \mathfrak{R}_{0}(P)$ satisfying $\mathfrak{q}_{1}(\alpha) \neq(1)$ let us arrange the prime ideal divisors $\mathfrak{p}$ of $F(\alpha)$ with $\mathfrak{p} \nmid \theta_{0}$ according to their norms. The ordering of ideals with equal norms is irrelevant. We now choose an integer $m=m(\alpha) \geq 1$ in such a way that

$$
N \mathfrak{g}(\alpha, m):=\prod_{\substack{j=1 \\ \mathfrak{p}_{j} \mid F(\alpha), \mathfrak{p}_{j} \nmid \theta_{0}}}^{m} N \mathfrak{p}_{j} \leq P^{1 / 4}, \quad \text { but } \prod_{\substack{j=1 \\ \mathfrak{p}_{j} \mid F(\alpha), \mathfrak{p}_{j} \nmid \theta_{0}}}^{m+1} N \mathfrak{p}_{j}>P^{1 / 4} .
$$

Remember that, in view of $\mathfrak{q}_{1}(\alpha) \neq(1)$, there is a prime ideal factor $\mathfrak{p}$ of $F(\alpha)$ with $N \mathfrak{p} \leq Z \leq P^{1 / 4}$.

Let us consider separately the sums

$$
\sum_{\substack{\alpha \in \mathfrak{R}_{0}(P) \\ N \mathfrak{g}(\alpha, m) \leq P^{1 / 6}}}^{\prime} H(F(\alpha)) \text { and } \sum_{\substack{\alpha \in \mathfrak{R}_{0}(P) \\ P^{1 / 6}<N \mathfrak{g}(\alpha, m) \leq P^{1 / 4}}}^{\prime} H(F(\alpha)) .
$$

In the first case we note that, by our choice of $m$, every prime ideal divisor $\mathfrak{p}_{j}$ of $F(\alpha)$ with $\mathfrak{p}_{j} \nmid \theta_{0}$ and $j>m$ satisfies

$$
N \mathfrak{p}_{j}>P^{1 / 4} \cdot N \mathfrak{g}(\alpha, m)^{-1} \geq P^{1 / 12} .
$$

Moreover, we obtain, in view of $H(\mathfrak{p}) \leq e$,

$$
H\left(\prod_{\substack{\mathfrak{p}^{l} \| F(\alpha) \\ \mathfrak{p} \mid \theta_{0}}} \mathfrak{p}^{l} \prod_{\substack{\mathfrak{p}_{j}^{l} \| F(\alpha) \\ j>m}} \mathfrak{p}_{j}^{l}\right) \ll \exp \left\{\sum_{\substack{\mathfrak{p}_{j} \mid F(\alpha) \\ j>m}} 1\right\} \ll \exp \{12 g\} .
$$

This leads us to the estimate

$$
\sum_{\substack{\alpha \in \mathfrak{R}_{0}(P) \\ \mathfrak{g}(\alpha, m) \leq P^{1 / 6}}}^{\prime} H(F(\alpha)) \ll \sum_{\substack{N \mathfrak{a} \leq P^{1 / 6} \\\left(\mathfrak{a}, \theta_{0}\right)=1}} \mu(\mathfrak{a})^{2} H(\mathfrak{a}) \sum_{\substack{\alpha \in \mathfrak{R}_{0}(P) \\ F(\alpha) \equiv 0 \bmod \mathfrak{a} \\ \mathfrak{p}|F(\alpha) \Rightarrow \mathfrak{p}| \mathfrak{a} \theta_{0} \text { or } N \mathfrak{p}>P^{1 / 12}}}
$$


If now we make use of Lemma 4 , we are able to conclude that

$$
\begin{aligned}
\sum_{\substack{\alpha \in \mathfrak{R}_{0}(P) \\
N \mathfrak{g}(\alpha, m) \leq P^{1 / 6}}}^{\prime} H(F(\alpha)) \\
\ll \frac{P}{\log P} \sum_{\substack{N \mathfrak{a} \leq P^{1 / 6} \\
\left(\mathfrak{a}, \theta_{0}\right)=1}} \mu(\mathfrak{a})^{2} H(\mathfrak{a}) \frac{L(\mathfrak{a})}{N \mathfrak{a}} \prod_{\mathfrak{p} \mid \mathfrak{a}}\left(1-\frac{L(\mathfrak{p})}{N \mathfrak{p}}\right)^{-1} .
\end{aligned}
$$

By standard calculations it follows at once from (11), (12) and (35) that the expression on the right-hand side of (37) has the desired order, namely

$$
\begin{aligned}
& \ll P(\log P)^{-1} \prod_{\substack{N \mathfrak{p} \leq P \\
\mathfrak{p} \nmid \theta_{0}}}\left(1+H(\mathfrak{p}) \frac{L(\mathfrak{p})}{N \mathfrak{p}-L(\mathfrak{p})}\right) \\
& \ll P(\log P)^{-1} \exp \left\{\sum_{N \mathfrak{p} \leq P}\left(H(\mathfrak{p}) \frac{L(\mathfrak{p})}{N \mathfrak{p}}+\frac{c_{23}}{N \mathfrak{p}^{2}}\right)\right\} \\
& \ll P \exp \left\{\sum_{N \mathfrak{p} \leq Z}(H(\mathfrak{p})-1) \frac{L(\mathfrak{p})}{N \mathfrak{p}}\right\} \\
& \ll P \exp \left\{\frac{e}{\log Z} \sum_{N \mathfrak{p} \leq Z} L(\mathfrak{p}) \frac{\log N \mathfrak{p}}{N \mathfrak{p}}\right\} \ll P .
\end{aligned}
$$

In order to attack the second sum in (36) we first note that, by (15) and (35),

$$
\prod_{\substack{j>m \\ \mid F(\alpha), \mathfrak{p}_{j} \nmid \theta_{0}}} H\left(\mathfrak{p}_{j}\right) \leq \exp \left\{\sum_{\substack{j>m \\ \mathfrak{p} \mid F(\alpha), \mathfrak{p}_{j} \nmid \theta_{0}}} 1\right\} \leq \exp \left\{(g+1) \frac{\log P}{\log N \mathfrak{p}_{m}}\right\} .
$$

Moreover,

$$
P^{1 / 6}<N \mathfrak{g}(\alpha, m) \leq N \mathfrak{p}_{m}^{v(\mathfrak{g}(\alpha, m))} \leq N \mathfrak{p}_{m}^{c_{24} \cdot \frac{\log P}{\log \log P}}
$$

Using these facts we find with $c_{25}=\left(6 c_{24}\right)^{-1}$ that

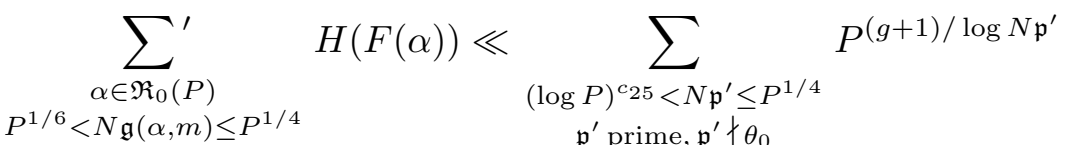

$$
\begin{aligned}
& \times \sum_{\substack{P^{1 / 6}<N \mathfrak{a} \leq P^{1 / 4} \\
\left(\mathfrak{a}, \theta_{0}\right)=1, \mathfrak{p}^{\prime}|\mathfrak{a} \\
\mathfrak{p}| \mathfrak{a} \Rightarrow N \mathfrak{p} \leq N \mathfrak{p}^{\prime}}} \mu(\mathfrak{a})^{2} H(\mathfrak{a}) \sum_{\begin{array}{c}
\alpha \in \Re_{0}(P) \\
F(\alpha) \equiv 0 \bmod \mathfrak{a} \\
\mathfrak{p}|F(\alpha) \Rightarrow \mathfrak{p}| \mathfrak{a} \theta_{0} \text { or } N \mathfrak{p}>N \mathfrak{p}^{\prime}-1
\end{array}} 1 .
\end{aligned}
$$


An application of Lemma 4 now leads us to the estimate

$$
\begin{gathered}
\sum_{\substack{\alpha \in \mathfrak{R}_{0}(P) \\
P^{1 / 6}<N \mathfrak{g}(\alpha, m) \leq P^{1 / 4}}}^{\prime} H(F(\alpha)) \ll P \sum_{\substack{c_{26} \leq N \mathfrak{p}^{\prime} \leq P^{1 / 4} \\
\mathfrak{p}^{\prime} \operatorname{prime}, \mathfrak{p}^{\prime} \nmid \theta_{0}}} \frac{P^{(g+1) / \log N \mathfrak{p}^{\prime}}}{\log N \mathfrak{p}^{\prime}} \\
\times \sum_{\substack{P^{1 / 6}<N \mathfrak{a} \leq P^{1 / 4} \\
\left(\mathfrak{a}, \theta_{0}\right)=1, \mathfrak{p}^{\prime}|\mathfrak{a} \\
\mathfrak{p}| \mathfrak{a} \Rightarrow N \mathfrak{p} \leq N \mathfrak{p}^{\prime}}} \mu(\mathfrak{a})^{2} H(\mathfrak{a}) \frac{L(\mathfrak{a})}{N \mathfrak{a}} \prod_{\mathfrak{p} \mid \mathfrak{a}}\left(1-\frac{L(\mathfrak{p})}{N \mathfrak{p}}\right)^{-1} .
\end{gathered}
$$

Using the abbreviation

$$
u_{0}=12(g+1) / \log N \mathfrak{p}^{\prime},
$$

the innermost sum in (38) gives, in view of (11), a contribution of order

$$
\begin{aligned}
& \leq \frac{H\left(\mathfrak{p}^{\prime}\right) L\left(\mathfrak{p}^{\prime}\right)}{N \mathfrak{p}^{\prime}-L\left(\mathfrak{p}^{\prime}\right)} \sum_{\substack{P^{1 / 6} / N \mathfrak{p}^{\prime}<N \mathfrak{b} \leq P^{1 / 4} / N \mathfrak{p}^{\prime} \\
\left(\mathfrak{b}, \theta_{0}\right)=1 \\
\mathfrak{p} \mid \mathfrak{b} \Rightarrow N \mathfrak{p} \leq N \mathfrak{p}^{\prime}}} \mu(\mathfrak{b})^{2} H(\mathfrak{b}) \frac{L(\mathfrak{b})}{N \mathfrak{b}} \prod_{\mathfrak{p} \mid \mathfrak{b}}\left(1-\frac{L(\mathfrak{p})}{N \mathfrak{p}}\right)^{-1} \\
& \ll\left(\frac{N \mathfrak{p}^{\prime}}{P^{1 / 6}}\right)^{u_{0}} \frac{1}{N \mathfrak{p}^{\prime}} \sum_{\substack{\left(\mathfrak{b}, \theta_{0}\right)=1 \\
\mathfrak{p} \mid \mathfrak{b} \Rightarrow N \mathfrak{p} \leq N \mathfrak{p}^{\prime}}} \mu(\mathfrak{b})^{2} H(\mathfrak{b}) L(\mathfrak{b}) N \mathfrak{b}^{u_{0}-1} \prod_{\mathfrak{p} \mid \mathfrak{b}}\left(1-\frac{L(\mathfrak{p})}{N \mathfrak{p}}\right)^{-1} \\
& \ll\left(N \mathfrak{p}^{\prime}\right)^{u_{0}-1} P^{-u_{0} / 6} \prod_{\substack{N \mathfrak{p} \leq N \mathfrak{p}^{\prime} \\
\mathfrak{p} \nmid \theta_{0}}}\left(1+\frac{H(\mathfrak{p}) L(\mathfrak{p})}{N \mathfrak{p}-L(\mathfrak{p})} N \mathfrak{p}^{u_{0}}\right) \\
& \ll\left(N \mathfrak{p}^{\prime}\right)^{-1} P^{-u_{0} / 6} \exp \left\{\sum_{\substack{N \mathfrak{p} \leq N \mathfrak{p}^{\prime} \\
\exp ^{\prime}}} \frac{H(\mathfrak{p}) L(\mathfrak{p})}{\left.N \mathfrak{p}^{1-u_{0}}\right\} .}\right.
\end{aligned}
$$

Moreover, by (12),

$$
\sum_{N \mathfrak{p} \leq N \mathfrak{p}^{\prime}} \frac{H(\mathfrak{p}) L(\mathfrak{p})}{N \mathfrak{p}}\left(N \mathfrak{p}^{u_{0}}-1\right) \ll u_{0} \sum_{N \mathfrak{p} \leq N \mathfrak{p}^{\prime}} \frac{L(\mathfrak{p})}{N \mathfrak{p}} \log N \mathfrak{p} \ll 1 .
$$

Returning to (38), these results yield, in view of (12), (35) and $e^{u} \geq u$ for $u \geq 0$,

$$
\begin{aligned}
& \sum_{\substack{\alpha \in \mathfrak{R}_{0}(P) \\
P^{1 / 6}<N \mathfrak{g}(\alpha, m) \leq P^{1 / 4}}}^{\prime} H(F(\alpha)) \\
& \ll P \sum_{N \mathfrak{p}^{\prime} \leq P} \frac{P^{-(g+1) / \log N \mathfrak{p}^{\prime}}}{N \mathfrak{p}^{\prime}} \exp \left\{\sum_{N \mathfrak{p} \leq N \mathfrak{p}^{\prime}} \frac{(H(\mathfrak{p})-1) L(\mathfrak{p})}{N \mathfrak{p}}\right\} \\
& \ll P(\log P)^{-1} \sum_{N \mathfrak{p} \leq P} \frac{\log N \mathfrak{p}}{N \mathfrak{p}} \exp \left\{\frac{e}{\log Z} \sum_{N \mathfrak{p} \leq Z} L(\mathfrak{p}) \frac{\log N \mathfrak{p}}{N \mathfrak{p}}\right\} \ll P .
\end{aligned}
$$


If we combine this with (31), (32), (33) and (34), we arrive readily at

$$
\begin{aligned}
\sum_{\substack{\alpha \in \mathfrak{R}_{0}(P) \\
N \mathfrak{q}_{1}(\alpha)>P^{1 / 3}}} \Delta(F(\alpha))^{t} & \ll_{t} P^{1 / 2}(\log P)^{2^{2 g t-1}}\left\{P^{\frac{1}{2}-\frac{1}{36 g}}+P^{\frac{1}{2}-\frac{1}{18 g \log Z}}\right\} \\
& \ll_{t} P(\log P)^{4^{g t}-1 /(18 g q(t))}
\end{aligned}
$$

where, for the last step, we have used (29). This is acceptable in Theorem 2 if $q(t) \leq 4^{-g t} /(18 g)$, as we henceforth assume.

Thus our problem has been reduced to that of establishing a suitable bound for the expression

$$
\sum_{\substack{\alpha \in \mathfrak{R}_{0}(P) \\ N \mathfrak{q}_{1}(\alpha) \leq P^{1 / 3}}} \Delta(F(\alpha))^{t},
$$

where $\mathfrak{q}_{1}(\alpha)=(1)$ if $(F(\alpha))=(1)$. We start from the obvious inequality

$$
\Delta(F(\alpha)) \leq \Delta\left(\mathfrak{q}_{1}(\alpha)\right) \sum_{\mathfrak{a} \mid \mathfrak{q}_{2}(\alpha)} 1,
$$

so that, making also use of (24),

$$
\begin{aligned}
\sum_{\substack{\alpha \in \mathfrak{R}_{0}(P) \\
N \mathfrak{q}_{1}(\alpha) \leq P^{1 / 3}}} \Delta(F(\alpha))^{t} \leq & \sum_{\substack{\alpha \in \mathfrak{R}_{0}(P) \\
N \mathfrak{q}_{1}(\alpha) \leq P^{1 / 3}}} \Delta\left(\mathfrak{q}_{1}(\alpha)\right)^{t}\left(\sum_{\mathfrak{a} \mid \mathfrak{q}_{2}(\alpha)} 1\right)^{t} \\
& \sum_{t} \sum_{\substack{\alpha \in \mathfrak{R}_{0}(P) \\
N \mathfrak{q}_{1}(\alpha) \leq P^{1 / 3}}} \Delta\left(\mathfrak{q}_{1}(\alpha)\right)^{t} \\
& \times \sum_{\substack{\mathfrak{a} \mid \mathfrak{q}_{2}(\alpha) \\
N \mathfrak{a} \leq N \mathfrak{q}_{2}(\alpha)^{1 /(3 g+1)}}}\left(\sum_{\mathfrak{c} \mid \mathfrak{a}} 1\right)^{(3 g+1) t} .
\end{aligned}
$$

For $\alpha \in \mathfrak{R}_{0}(P)$ we have from (15),

$$
N \mathfrak{q}_{2}(\alpha)^{1 /(3 g+1)} \leq|N F(\alpha)|^{1 /(3 g+1)} \leq P^{1 / 3} .
$$

This leads us immediately to

$$
\begin{aligned}
& \sum_{\substack{\alpha \in \mathfrak{R}_{0}(P) \\
N \mathfrak{q}_{1}(\alpha) \leq P^{1 / 3}}} \Delta(F(\alpha))^{t} \\
& \ll_{t} \sum_{\substack{N \mathfrak{a} \leq P^{1 / 3} \\
\mathfrak{p} \mid \mathfrak{a} \Rightarrow N \mathfrak{p} \leq Z}} \Delta(\mathfrak{a})^{t} \sum_{\substack{N \mathfrak{b} \leq P^{1 / 3} \\
\mathfrak{p} \mid \mathfrak{b} \Rightarrow N \mathfrak{p}>Z}}\left(\sum_{\mathfrak{c} \mid \mathfrak{b}} 1\right)^{(3 g+1) t} \sum_{\begin{array}{c}
\alpha \in \mathfrak{R}_{0}(P) \\
F(\alpha) \equiv 0 \bmod \mathfrak{a} \mathfrak{b} \\
\mathfrak{p}|F(\alpha) \Rightarrow \mathfrak{p}| \mathfrak{a} \text { or } N \mathfrak{p}>Z
\end{array}} 1 .
\end{aligned}
$$


We are now again in a position to apply Lemma 4, and we obtain, in view of $(29)$,

$$
\begin{aligned}
\sum_{\substack{\alpha \in \mathfrak{R}_{0}(P) \\
N \mathfrak{q}_{1}(\alpha) \leq P^{1 / 3}}} \Delta(F(\alpha))^{t} \ll_{t} & \frac{P \log \log P}{\log P} \sum_{N \mathfrak{a} \leq P} \Delta(\mathfrak{a})^{t} \frac{L(\mathfrak{a})}{N \mathfrak{a}} \\
& \times \prod_{\substack{\mathfrak{p} \mid \mathfrak{a} \\
\mathfrak{p} \nmid \theta_{0}}}\left(1-\frac{L(\mathfrak{p})}{N \mathfrak{p}}\right)^{-1} \sum_{\substack{N \mathfrak{b} \leq P \\
\mathfrak{p} \mid \mathfrak{b} \Rightarrow N \mathfrak{p}>Z}} \frac{L(\mathfrak{b})}{N \mathfrak{b}} \\
& \times\left(\sum_{\mathfrak{c} \mid \mathfrak{b}} 1\right)^{(3 g+1) t} \prod_{\substack{\mathfrak{p} \mid \mathfrak{b} \\
\mathfrak{p} \nmid \theta_{0}}}\left(1-\frac{L(\mathfrak{p})}{N \mathfrak{p}}\right)^{-1} .
\end{aligned}
$$

It is easy to check (cf. [8], Lemma 4.2) that

$$
\prod_{\substack{\mathfrak{p} \mid \mathfrak{d} \\ \mathfrak{p} \nmid \theta_{0}}}\left(1-\frac{L(\mathfrak{p})}{N \mathfrak{p}}\right)^{-1} \ll \log \log 3 N \mathfrak{d},
$$

so that finally

$$
\begin{aligned}
\sum_{\substack{\alpha \in \mathfrak{R}_{0}(P) \\
N \mathfrak{q}_{1}(\alpha) \leq P^{1 / 3}}} \Delta(F(\alpha))^{t} & \ll_{t} \frac{P(\log \log P)^{3}}{\log P} \sum_{N \mathfrak{a} \leq P} \Delta(\mathfrak{a})^{t} \frac{L(\mathfrak{a})}{N \mathfrak{a}} \\
& \times \prod_{Z<N \mathfrak{p} \leq P}\left(\sum_{j=0}^{\infty} \frac{L\left(\mathfrak{p}^{j}\right)}{N \mathfrak{p}^{j}}(j+1)^{(3 g+1) t}\right) \\
& \ll_{t} P(\log P)^{-1+o(1)} \sum_{N \mathfrak{a} \leq P} \Delta(\mathfrak{a})^{t} \frac{L(\mathfrak{a})}{N \mathfrak{a}} \quad \text { as } P \rightarrow \infty .
\end{aligned}
$$

For the last step we have only to observe that, by (12) and our choice of $Z$ in $(29)$,

$$
\begin{aligned}
\prod_{Z<N \mathfrak{p} \leq P}\left(\sum_{j=0}^{\infty} \frac{L\left(\mathfrak{p}^{j}\right)}{N \mathfrak{p}^{j}}(j+1)^{(3 g+1) t}\right) & \ll_{t} \exp \left\{2^{(3 g+1) t} \sum_{Z<N \mathfrak{p} \leq P} \frac{L(\mathfrak{p})}{N \mathfrak{p}}\right\} \\
& \ll_{t}(\log \log P)^{2^{(3 g+1) t}}
\end{aligned}
$$

To complete the proof of Theorem 2 it only remains to apply Lemma 5 .

Proof of Theorem 1. For $b, s>0$ it follows from (21)-(23) that 


$$
N(P) \gg P(\log P)^{-b}-(\log P)^{-b(s+1)} \sum_{\alpha \in \Re_{0}(P)} \Delta(F(\alpha))^{1+s} .
$$

An appeal to Theorem 2 leads us to the estimate

$$
\begin{aligned}
N(P) & \gg P(\log P)^{-b}-P(\log P)^{2^{s+1}-s-b(s+1)-2+o(1)} \\
& \gg P(\log P)^{-b}-P(\log P)^{2^{s+1}-(b+1)(s+1)-1+o(1) .}
\end{aligned}
$$

Since

$$
\frac{2\left(2^{s}-1\right)}{s}=\frac{2}{s}\left(e^{s \log 2}-1\right)>2 \log 2>1
$$

we have

$$
N(P) \gg P(\log P)^{-b} \quad \text { if } b>\frac{2\left(2^{s}-1\right)}{s}-1 .
$$

For $b>\log 4-1$ we choose

$$
s=\frac{1}{\log 2} \log \frac{b+1}{\log 4}>0 .
$$

This is admissible because of

$$
\frac{2\left(2^{s}-1\right)}{s}-1<\frac{2 \cdot 2^{s} \cdot s \cdot \log 2}{s}-1=2^{s} \log 4-1=b .
$$

Theorem 1 now follows in view of (6).

\section{References}

[1] P. Erdős, On the greatest prime factor of $\prod_{k=1}^{x} f(k)$, J. London Math. Soc. 27 (1952), 379-384.

[2] P. Erdős and A. Schinzel, On the greatest prime factor of $\prod_{k=1}^{x} f(k)$, Acta Arith. 55 (1990), 191-200.

[3] R. R. Hall and G. Tenenbaum, Divisors, Cambridge University Press, Cambridge, 1988.

[4] J. G. Hinz, Some applications of sieve methods in algebraic number fields, Manuscripta Math. 48 (1984), 117-137.

[5] B. Landreau, A new proof of a theorem of van der Corput, Bull. London Math. Soc. 21 (1989), 366-368.

[6] T. Nagell, Généralisation d'un théorème de Tchebycheff, J. Math. Pures Appl. (8) 4 (1921), 343-356.

[7] G. J. Rieger, Verallgemeinerung der Siebmethode von A. Selberg auf algebraische Zahlkörper. III, J. Reine Angew. Math. 208 (1961), 79-90.

[8] H. Sarges, Eine Anwendung des Selbergschen Siebes auf algebraische Zahlkörper, Acta Arith. 28 (1976), 433-455.

[9] C. L. Siegel, Additive Theorie der Zahlkörper. II, Math. Ann. 88 (1923), 184-210. 
[10] G. Tenenbaum, Sur une question d'Erdős et Schinzel, in: A Tribute to Paul Erdős, A. Baker, B. Bollobás, A. Hajnal (eds.), Cambridge University Press, Cambridge, 1990, 405-443.

[11] —, Sur une question d'Erdös et Schinzel. II, Invent. Math. 99 (1990), 215-224.

DEPARTMENT OF MATHEMATICS

UNIVERSITY OF MARBURG

HANS-MEERWEIN-STRASSE, LAHNBERGE

D-35032 MARBURG, GERMANY

Received on 8.1.1995

and in revised form on 15.7.1995 